\title{
Single-basined choice
}

Citation for published version (APA):

Bossert, W., \& Peters, H. J. M. (2013). Single-basined choice. Maastricht University, Graduate School of Business and Economics. GSBE Research Memoranda No. 030 https://doi.org/10.26481/umagsb.2013030

Document status and date:

Published: 01/01/2013

DOI:

10.26481/umagsb.2013030

Document Version:

Publisher's PDF, also known as Version of record

\section{Please check the document version of this publication:}

- A submitted manuscript is the version of the article upon submission and before peer-review. There can be important differences between the submitted version and the official published version of record.

People interested in the research are advised to contact the author for the final version of the publication, or visit the DOI to the publisher's website.

- The final author version and the galley proof are versions of the publication after peer review.

- The final published version features the final layout of the paper including the volume, issue and page numbers.

Link to publication

\footnotetext{
General rights rights.

- You may freely distribute the URL identifying the publication in the public portal. please follow below link for the End User Agreement:

www.umlib.nl/taverne-license

Take down policy

If you believe that this document breaches copyright please contact us at:

repository@maastrichtuniversity.nl

providing details and we will investigate your claim.
}

Copyright and moral rights for the publications made accessible in the public portal are retained by the authors and/or other copyright owners and it is a condition of accessing publications that users recognise and abide by the legal requirements associated with these

- Users may download and print one copy of any publication from the public portal for the purpose of private study or research.

- You may not further distribute the material or use it for any profit-making activity or commercial gain

If the publication is distributed under the terms of Article $25 \mathrm{fa}$ of the Dutch Copyright Act, indicated by the "Taverne" license above, 


\section{Maastricht University}

Walter Bossert, Hans Peters

Single-basined choice

RM/13/030

\section{GSBE}

Maastricht University School of Business and Economics

Graduate School of Business and Economics

P.O Box 616

NL- 6200 MD Maastricht

The Netherlands 


\title{
Single-basined choice*
}

\author{
WALTER BOSSERT \\ Department of Economics and CIREQ, University of Montreal \\ P.O. Box 6128, Station Downtown \\ Montreal QC H3C 3J7, Canada \\ walter.bossert@umontreal.ca \\ and \\ HANS Peters \\ Department of Quantitative Economics, Maastricht University \\ P.O. Box 616 \\ 6200 MD Maastricht, The Netherlands \\ h.peters@maastrichtuniversity.nl
}

This version: June 3, 2013

\begin{abstract}
Single-basined preferences generalize single-dipped preferences by allowing for multiple worst elements. These preferences have played an important role in areas such as voting, strategy-proofness and matching problems. We examine the notion of singlebasinedness in a choice-theoretic setting. In conjunction with independence of irrelevant alternatives, single-basined choice implies a structure that conforms to the motivation underlying our definition. We also establish the consequences of requiring single-basined choice correspondences to be upper semicontinuous, and of the revealed preference relation to be Suzumura consistent. Journal of Economic Literature Classification Nos.: D11, D71.
\end{abstract} Keywords. Single-basinedness, choice correspondences, independence of irrelevant alternatives, upper semicontinuity, Suzumura consistency.

* Financial support from CIREQ, the Research School METEOR of Maastricht University, the Dutch Science Foundation NWO (grant no. 040.11.320), the Fonds de Recherche sur la Société et la Culture of Québec, and the Social Sciences and Humanities Research Council of Canada is gratefully acknowledged. 


\section{Introduction}

Single-peakedness has a long history in economic theory. It goes back as far as Black (1948) who shows that if preferences are restricted to those that are single-peaked, then the majority rule generates transitive social preferences. Other important contributions include Inada (1969) and Sen (1970) who provide related value restrictions that focus on single-peaked preference profiles. See also Moulin (1980) and Sprumont (1991) for applications in the context of strategy-proofness. There is no need to restrict the notion of single-peakedness to a single dimension. Generalizations to higher dimensions are employed, for instance, by Barberà, Gul and Stacchetti (1993), Barberà and Jackson (1994), Dutta, Peters and Sen (2002), Ehlers and Storcken (2008) and Le Breton and Weymark (2011). Ballester and Haeringer (2011) characterize one-dimensional single-peaked preference profiles by providing necessary and sufficient conditions for the existence of a single ranking such that all preferences in the profile are single-peaked with respect to this ranking.

Single-peakedness and its generalization single-plateauedness are analyzed in a choicetheoretic setting by Bossert and Peters (2009, 2013). The notion of single-peakedness is too restrictive if choices are permitted to be multi-valued. This generalization is applied by Moulin (1984), Berga (1998), Ehlers (2002a), Barberà (2007) and Berga and Moreno (2009), among others.

The natural counterpart of single-peakedness is single-dippedness, where preferences are such that there is a single 'dip' rather than a single peak, and we refer to its generalization to environments that permit multi-valuedness as single-basinedness. Single-dipped preferences frequently appear when a public bad is to be located and individuals are assumed to prefer a larger distance from the bad to being closer to its chosen location. The relevant literature includes Kunreuther and Kleindorfer (1986), Klaus, Peters and Storcken (1997), Peremans and Storcken (1999), Klaus (2001), Ehlers (2002b), Lescop (2007), Besfamille and Lozachmeur (2010), Barberà, Berga and Moreno (2012), Öztürk, Peters and Storcken (2012, 2013) and Manjunath (2013).

The purpose of this paper is to provide a choice-theoretic basis for single-basined preferences. We work within a Euclidean space that can be of any (fixed) dimension and study choice correspondences that select a non-empty and compact subset of chosen elements from each non-empty, compact and convex subset of $\mathbb{R}^{n}$, the Euclidean $n$-dimensional space. As is common in this type of literature, we concentrate on choice correspondences that satisfy independence of irrelevant alternatives, a contraction consistency condition that is necessary (but, in general, not sufficient) for the rationalizability of a choice correspondence by 
an ordering; see, for instance, Richter $(1966,1971)$. In view of the typical applications of single-dippedness and single-basinedness, this focus is suitable. We additionally impose the condition of single-basinedness on a choice correspondence: this condition says that if $x$ is revealed preferred to $y$, then also all points on the straight line through $x$ and $y$, further away from $y$ than $x$, are revealed preferred to $y$. We then show that such a choice correspondence either has a basin, which is a (convex) set of worst points, or it does not have a basin, in which case it always picks boundary points from a choice set. Also, if the choice correspondence is upper semicontinuous and has a basin, then this basin is closed. Finally, we show that adding upper semicontinuity and Suzumura consistency results in the choice correspondence assigning the maximizers of a quasi-convex function.

\section{Independent choice correspondences}

Suppose $n \in \mathbb{N}$ is fixed and define $\mathcal{C}=\left\{C \subseteq \mathbb{R}^{n} \mid C\right.$ is non-empty, compact and convex $\}$. A choice correspondence is a mapping $\varphi: \mathcal{C} \rightarrow \mathbb{R}^{n}$ such that $\emptyset \neq \varphi(C) \subseteq C$ and $\varphi(C)$ is compact for all $C \in \mathcal{C}$.

The direct revealed preference relation $R_{\varphi}$ of $\varphi$ is defined as follows. For all $x, y \in \mathbb{R}^{n}$,

$$
x R_{\varphi} y \Leftrightarrow \text { there exists } C \in \mathcal{C} \text { such that } x \in \varphi(C) \text { and } y \in C \text {. }
$$

The asymmetric part of $R_{\varphi}$ is denoted by $P_{\varphi}$, and $I_{\varphi}$ is the symmetric part of $R_{\varphi}$.

A generalized version of Samuelson's (1938) weak axiom of revealed preference can be stated as follows (see Bossert and Suzumura, 2010, p. 17, for a discussion and alternative formulations).

Weak axiom of revealed preference. For all $C \in \mathcal{C}$ and for all $x, y \in C$, if $x R_{\varphi} y$ and $y \in \varphi(C)$, then $x \in \varphi(C)$.

In our framework, the weak axiom of revealed preference is equivalent to independence of irrelevant alternatives, which is a contraction-consistency condition imposed on a choice correspondence. It is often referred to as Arrow's choice axiom (see Arrow, 1959) but, as Shubik (1982, pp. 420-421 and p. 423, footnote 2) remarks, the axiom already appears in 1950 in an informal note authored by Nash. A version for single-valued choice is due to Nash (1950) in the context of axiomatic bargaining theory.

Independence of irrelevant alternatives. For all $C, D \in \mathcal{C}$, if $D \subseteq C$ and $D \cap \varphi(C) \neq \emptyset$, then $\varphi(D)=D \cap \varphi(C)$. 
For future reference, we note that, because our domain $\mathcal{C}$ is closed under intersection (that is, for all $C, D \in \mathcal{C}$, the intersection $C \cap D$ is also in $\mathcal{C}$ whenever this intersection is non-empty), the weak axiom of revealed preference is equivalent to independence of irrelevant alternatives; see Hansson (1968) for a generalization of this observation. We state this known result without proving it here; an explicit proof is provided in Bossert and Peters (2013).

Lemma 1 A choice correspondence $\varphi: \mathcal{C} \rightarrow \mathbb{R}^{n}$ satisfies independence of irrelevant alternatives if and only if $\varphi$ satisfies the weak axiom of revealed preference.

As is well-known, the weak axiom of revealed preference implies independence of irrelevant alternatives even if the domain of a choice correspondence is not closed under intersection. As a consequence of Lemma 1, we can use independence of irrelevant alternatives and the weak axiom of revealed preference interchangeably.

\section{Single-basined choice correspondences}

For distinct $x, y \in \mathbb{R}^{n},[x, y, \rightarrow)$ is the half-line through $y$ starting at $x$ and $[x, y]$ is the line segment with end points $x$ and $y$. The (relatively) half-open sets $[x, y)$ and $(x, y]$, and the (relatively) open set $(x, y)$ are defined analogously in the usual way. The boundary of $C \in \mathcal{C}$ is denoted by $b d(C)$ and the interior of $C$ is $\operatorname{int}(C)$. The convex hull of $C$ is $\operatorname{conv}(C)$ and the closure of a subset $D$ of $\mathbb{R}^{n}$ is denoted by $\operatorname{cl}(D)$. Convergence of a sequence of sets in $\mathcal{C}$ is defined in terms of the Hausdorff metric for compact subsets of $\mathbb{R}^{n}$. The function $d: \mathbb{R}^{n} \times \mathbb{R}^{n} \rightarrow \mathbb{R}_{+}$denotes the Euclidean distance in $\mathbb{R}^{n}$.

Single-basinedness of a choice correspondence is defined as follows.

Single-basinedness. For all distinct $x, y \in \mathbb{R}^{n}$, if $x R_{\varphi} y$, then $z R_{\varphi} y$ for all $z \in[y, x, \rightarrow)$ with $z \notin[y, x)$.

Thus, single-basinedness demands that if a point $x$ is directly revealed preferred to another point $y$, then any point $z$ that is located on the half-line starting at $y$ and passing through $x$ and that is, moreover, at least as far away from $y$ as $x$, is directly revealed preferred to $y$.

We conclude this section with two lemmas that are used in proving our theorems.

Lemma 2 Let the choice correspondence $\varphi: \mathcal{C} \rightarrow \mathbb{R}^{n}$ satisfy independence of irrelevant alternatives and single-basinedness, and let $C \in \mathcal{C}$. If $\varphi(C) \cap \operatorname{int}(C) \neq \emptyset$, then $\varphi(C)=C$. 
Proof. We need to prove that $C \subseteq \varphi(C)$. Let $x \in \varphi(C) \cap \operatorname{int}(C)$. For any $y \in C \backslash\{x\}$, there exists $z \in[y, x, \rightarrow) \cap C$ such that $x \in(y, z)$ because $x \in \operatorname{int}(C)$. We thus have $x R_{\varphi} z$ because $x \in \varphi(C)$ and $z \in C$. By single-basinedness, it follows that $y R_{\varphi} z$. Thus, there exists $C^{\prime} \in \mathcal{C}$ such that $y \in \varphi\left(C^{\prime}\right)$ and $z \in C^{\prime}$. By independence of irrelevant alternatives, $y \in \varphi([y, z])$ and hence $y R_{\varphi} x$ because $x \in[y, z]$. Because $y \in C$ and $x \in \varphi(C)$, the weak axiom of revealed preference implies $y \in \varphi(C)$.

Lemma 3 Let the choice correspondence $\varphi: \mathcal{C} \rightarrow \mathbb{R}^{n}$ satisfy independence of irrelevant alternatives and single-basinedness, let $C \in \mathcal{C}$ and let $x, y, z \in C$ be such that $z \in(x, y)$. If $z \in \varphi(C)$, then $[x, y] \subseteq \varphi(C)$.

Proof. Suppose $x, y, z \in C$ and $z \in \varphi(C) \cap(x, y)$. Let $w \in[x, y] \backslash\{z\}$. Consider first the case $w \in[x, z)$. Clearly, there exists $v \in(z, y]$ because $z \in(x, y)$. We have $z R_{\varphi} v$ because $z \in \varphi(C)$ and $v \in[x, y] \subseteq C$. By single-basinedness, it follows that $w R_{\varphi} v$. Thus, there exists $C^{\prime} \in \mathcal{C}$ such that $w \in \varphi\left(C^{\prime}\right)$ and $v \in C^{\prime}$. By independence of irrelevant alternatives, $w \in \varphi([w, v])$ and hence $w R_{\varphi} z$ because $z \in[w, v]$. Because $w \in[x, y] \subseteq C$ and $z \in \varphi(C)$, the weak axiom of revealed preference implies $w \in \varphi(C)$. The proof for the case $w \in(z, y]$ is identical.

\section{Basins}

A set $B \subseteq \mathbb{R}^{n}$ is a basin for a choice correspondence $\varphi$ if $B \neq \emptyset$ and, for all $x \in B$ and for all $C \in \mathcal{C}$ such that $x \in C$,

$$
x \in \varphi(C) \Leftrightarrow C \subseteq B .
$$

If such a set $B$ exists, we say that the choice correspondence $\varphi$ has a basin.

As a first observation regarding the properties of a basin, we state the following lemma. It establishes that if a choice correspondence $\varphi$ satisfies single-basinedness and has a basin $B$, then $B$ must be a convex set.

Lemma 4 Let the choice correspondence $\varphi: \mathcal{C} \rightarrow \mathbb{R}^{n}$ satisfy single-basinedness and suppose that $\varphi$ has a basin $B$. Then $B$ is convex.

Proof. Let $x, y \in B$. We have to show that $[x, y] \subseteq B$. If $x \in \varphi([x, y])$ or $y \in \varphi([x, y])$, then $[x, y] \subseteq B$ follows from the definition of a basin. Otherwise, let $z \in \varphi([x, y]) \cap(x, y)$. Then $z R_{\varphi} x$ and, by single-basinedness, $y R_{\varphi} x$. By definition of $R_{\varphi}$, there exists $C \in \mathcal{C}$ such 
that $y \in \varphi(C)$ and $x \in C$. By definition of $B$, this implies $C \subseteq B$ and, hence, $[x, y] \subseteq B$ because $[x, y] \subseteq C$ by the convexity of $C$.

Next, we show that if a choice correspondence satisfies single-basinedness and has a basin, then this basin must be unique.

Lemma 5 Let the choice correspondence $\varphi: \mathcal{C} \rightarrow \mathbb{R}^{n}$ satisfy single-basinedness and suppose that $\varphi$ has a basin $B$ and a basin $B^{\prime}$. Then $B=B^{\prime}$.

Proof. By way of contradiction, suppose that $B$ and $B^{\prime}$ are basins for $\varphi$ and $B \neq B^{\prime}$. Without loss of generality, suppose there exists $x \in B \backslash B^{\prime}$.

Case (i). $B \cap B^{\prime} \neq \emptyset$. Let $y \in B \cap B^{\prime}$, which immediately implies that $y \neq x$. Because $x, y \in B$ and $B$ is convex by Lemma 4 , it follows that $[x, y] \subseteq B$. By definition of a basin, $\varphi([x, y])=[x, y]$. Because $y \in B^{\prime}, y \in[x, y]$ and $y \in \varphi([x, y])$, we obtain $[x, y] \subseteq B^{\prime}$ since $B^{\prime}$ is a basin. This contradicts our supposition that $x \notin B^{\prime}$.

Case (ii). $B \cap B^{\prime}=\emptyset$. Let $x \in B$ and $y \in B^{\prime}$. Then, again by definition of a basin, $\varphi([x, y]) \cap B=\varphi([x, y]) \cap B^{\prime}=\emptyset$. Let $z \in \varphi([x, y])$. Thus $z R_{\varphi} y$ by definition of $R_{\varphi}$, and hence $x R_{\varphi} y$ by single-basinedness. Let $C \in \mathcal{C}$ be such that $x \in \varphi(C)$ and $y \in C$. This implies $C \subseteq B$ because $B$ is a basin and thus $y \in B$, contradicting the supposition that $y \notin B$.

An immediate corollary of combining the above two lemmas is that if $\varphi$ satisfies singlebasinedness and has a basin, then this basin is unique and convex.

The following theorem illustrates that our definition of single-basinedness suitably captures a multi-valued generalization of single-dipped choice in the presence of irrelevant alternatives if $\varphi$ has a basin. The properties of such a choice correspondence reflect those associated with a unique set of 'worst' elements in the choice-theoretic sense. That is, (a) whenever a feasible set $C$ is contained in the basin $B$, the entire set $C$ is chosen. In all other cases (that is, whenever $C$ contains points in $\mathbb{R}^{n}$ that are not in $B$ ), (b) none of the points in $B$ are in $\varphi(C)$, and (c) only boundary points of $C$ are chosen. In addition, (d) there is a well-defined sense in which 'better' points are reached when we move away from $B$ along a half-line.

Theorem 1 Let the choice correspondence $\varphi: \mathcal{C} \rightarrow \mathbb{R}^{n}$ satisfy independence of irrelevant alternatives and single-basinedness and suppose that $\varphi$ has a basin $B$. Then $B$ is unique and convex and, for all $C \in \mathcal{C}$, 
(a) $C \subseteq B \Rightarrow \varphi(C)=C$;

(b) $C \nsubseteq B \Rightarrow \varphi(C) \cap B=\emptyset$;

(c) $C \nsubseteq B \Rightarrow \varphi(C) \subseteq b d(C)$;

(d) $C \nsubseteq B \Rightarrow[b, x, \rightarrow) \cap C \subseteq[b, x]$ for all $x \in \varphi(C)$ and for all $b \in B$.

Proof. (a) Suppose $C \subseteq B$. Because $B$ is a basin, we have $x \in \varphi(C)$ for all $x \in C$. This implies $C \subseteq \varphi(C)$ and, therefore, $\varphi(C)=C$.

(b) Suppose $C \nsubseteq B$. If $\varphi(C) \cap B \neq \emptyset$, it follows that $C \subseteq B$ by definition of a basin, a contradiction to our hypothesis. Thus, we must have $\varphi(C) \cap B=\emptyset$.

(c) Suppose $x \in \varphi(C) \cap \operatorname{int}(C)$. By (b), $x \notin B$. Since $x \in \operatorname{int}(C)$, we can take $b \in B$ and $y \in C$ such that $x \in(b, y)$. Then $x R_{\varphi} y$ by definition of $R_{\varphi}$, and single-basinedness implies $b R_{\varphi} y$. By definition of $R_{\varphi}$, there exists $C^{\prime} \in \mathcal{C}$ such that $b \in \varphi\left(C^{\prime}\right)$ and $y \in C^{\prime}$. Independence of irrelevant alternatives implies $b \in \varphi([b, y])$. Because $x \in[b, y]$, we obtain $b R_{\varphi} x$. By definition of a basin, $[b, x] \subseteq B$ and, thus, $x \in B$, a contradiction.

(d) Suppose there exists $y \in[b, x, \rightarrow) \cap C$ such that $y \notin[b, x]$. Thus, $x \in[b, y)$ and, because $x \in \varphi(C)$ and $y \in C$, we have $x R_{\varphi} y$. Single-basinedness implies $b R_{\varphi} y$ and the same argument as in the proof of (c) applies.

Note that the statements (a) and (b) in the above theorem are true whenever $\varphi$ has a basin; independence of irrelevant alternatives and single-basinedness are not required in the respective proofs. Furthermore, the uniqueness and the convexity of $B$ are not invoked in the proof of any part of the result.

\section{The case without a basin}

Even if a choice correspondence does not have a basin, independence of irrelevant alternatives and single-basinedness imply that the spirit of single-basined choice is respected. Of course, if $\varphi$ has no basin, then statements (a), (b) and (d) in Theorem 1 become vacuous. However, there is an analogue to part (c) the proof of which is more involved than one would expect. More precisely, we obtain the following theorem.

Theorem 2 Let the choice correspondence $\varphi: \mathcal{C} \rightarrow \mathbb{R}^{n}$ satisfy independence of irrelevant alternatives and single-basinedness. If $\varphi$ does not have a basin, then $\varphi(C) \subseteq b d(C)$ for all $C \in \mathcal{C}$. 
Proof. Suppose that there exists $C^{\prime} \in \mathcal{C}$ such that $\varphi\left(C^{\prime}\right) \cap \operatorname{int}\left(C^{\prime}\right) \neq \emptyset$. By Lemma 2, $\varphi\left(C^{\prime}\right)=C^{\prime}$. We now construct a set $B \subseteq \mathbb{R}^{n}$ and show that it is a basin for $\varphi$, which will complete the proof of the theorem. Let $b \in \operatorname{int}\left(C^{\prime}\right)$ (hence $b \in \varphi\left(C^{\prime}\right)$ ) and let $H$ be the set of all half-lines emanating from $b$. Consider any $h \in H$. Since $b \in \operatorname{int}\left(C^{\prime}\right)$, we can take $b^{\prime} \in\left(h \cap C^{\prime}\right) \backslash\{b\}$. Now $\varphi\left(C^{\prime}\right)=C^{\prime}$ implies $b^{\prime} R_{\varphi} b$ and hence $z R_{\varphi} b$ for all $z \in h$ by singlebasinedness. Furthermore, $b R_{\varphi} z$ for all $z \in h \cap C^{\prime}$. If $b R_{\varphi} z$ for all $z \in h$, define $B_{h}=h$. Otherwise let $\hat{z}_{h}=\arg \inf _{z}\left\{d(z, b) \mid z \in h\right.$ and $\left.z P_{\varphi} b\right\}$. If $\hat{z}_{h} P_{\varphi} b$, define $B_{h}=\left[b, \hat{z}_{h}\right)$; if $\hat{z}_{h} I_{\varphi} b$, define $B_{h}=\left[b, \hat{z}_{h}\right]$. Now define $B=\bigcup_{h \in H} B_{h}$. Because $b \in B, B$ is non-empty. It remains to be shown that, for all $C \in \mathcal{C}$ and for all $x \in B \cap C$,

$$
x \in \varphi(C) \Leftrightarrow C \subseteq B .
$$

(i) " $\Rightarrow$." Suppose $x \in \varphi(C)$. Let $y \in C$. We have to prove that $y \in B$. If $x=b$, we have $b R_{\varphi} y$ and, by definition of $B, y \in B$. If $y=x$ or $y=b$, the desired conclusion follows trivially because $b, x \in B$ by assumption. This leaves the case in which the three points $b$, $x$ and $y$ are pairwise distinct.

Consider $\Delta=\operatorname{conv}(\{b, x, y\})$. Because $x \in B$, the definition of $B$ implies $b R_{\varphi} x$. Because $x \in h$ for some $h \in H$, we have $x R_{\varphi} b$. Hence, $x I_{\varphi} b$. Because $x \in \varphi(C)$ and $y \in C$, it follows that $x R_{\varphi} y$. The following three cases cover all possibilities.

(i.1) $\varphi(\Delta) \cap\{b, x, y\} \neq \emptyset$. If $b \in \varphi(\Delta)$, it follows that $b R_{\varphi} y$ and thus $y \in B$ by definition of $B$. If $x \in \varphi(\Delta)$, the weak axiom of revealed preference implies $b \in \varphi(\Delta)$ because $b I_{\varphi} x$ and $b \in \Delta$ and, again, $y \in B$ follows. If $y \in \varphi(\Delta)$, the weak axiom of revealed preference implies $x \in \varphi(\Delta)$ because $x R_{\varphi} y$. Hence, $y \in B$ as just established.

(i.2) $\varphi(\Delta) \cap \operatorname{int}(\Delta) \neq \emptyset$. By Lemma $2, \varphi(\Delta)=\Delta$ and thus $b \in \varphi(\Delta)$ which brings us back to case (i.1).

(i.3) $\varphi(\Delta) \cap((b, x) \cup(b, y) \cup(x, y)) \neq \emptyset$. Lemma 3 immediately implies $\varphi(\Delta) \cap\{b, x, y\} \neq \emptyset$ and, again, we are back to case (i.1).

(ii) " $\Leftarrow$." Suppose $C \subseteq B$. We have to prove that $x \in \varphi(C)$. Because $x \in B$, the definition of $B$ implies $b R_{\varphi} x$. Because $x \in h$ for some $h \in H$, we have $x R_{\varphi} b$. Hence, $x I_{\varphi} b$. Let $y \in \varphi(C)$. Because $\varphi(C) \subseteq C \subseteq B$, this implies $y \in B$. Hence, by the same argument that was just used for $x$, we also have $y I_{\varphi} b$. If $x=y$, we are done. If $x=b$ or $y=b$, we have $x I_{\varphi} y$ and the weak axiom of revealed preference implies $x \in \varphi(C)$ because $x \in C$ and $y \in \varphi(C)$. It remains to consider the case in which the three points $b, x$ and $y$ are pairwise distinct.

Again, let $\Delta=\operatorname{conv}(\{b, x, y\})$. Because $x \in \varphi(C)$ and $y \in C$, it follows that $x R_{\varphi} y$. The following three cases cover all possibilities. 
(ii.1) $\varphi(\Delta) \cap\{b, x, y\} \neq \emptyset$. If $x \in \varphi(\Delta)$, it follows that $x R_{\varphi} y$ and thus $x \in \varphi(C)$ by the weak axiom of revealed preference. If $b \in \varphi(\Delta)$, the weak axiom of revealed preference implies $x \in \varphi(\Delta)$ because $x I_{\varphi} b$ and $b \in \Delta$ and, again, $x \in \varphi(C)$ follows. If $y \in \varphi(\Delta)$, the weak axiom of revealed preference implies $b \in \varphi(\Delta)$ because $b I_{\varphi} y$. Hence, $x \in \varphi(C)$ as just established.

(ii.2) $\varphi(\Delta) \cap \operatorname{int}(\Delta) \neq \emptyset$. By Lemma 2, $\varphi(\Delta)=\Delta$ and thus $x \in \varphi(\Delta)$ which brings us back to case (ii.1).

(ii.3) $\varphi(\Delta) \cap((b, x) \cup(b, y) \cup(x, y)) \neq \emptyset$. Lemma 3 immediately implies $\varphi(\Delta) \cap\{b, x, y\} \neq$ $\emptyset$ and, again, we are back to case (ii.1).

\section{Continuity}

In this section, we explore to what extent standard continuity properties may allow us to narrow down the class of single-basined choice correspondences discussed in the previous section.

Continuity. For all $C \in \mathcal{C}$ and for all sequences $\left\langle C^{i}\right\rangle_{i \in \mathbb{N}}$ with $C^{i} \in \mathcal{C}$ for all $i \in \mathbb{N}$, if $\lim _{i \rightarrow \infty} C^{i}=C$, then $\lim _{i \rightarrow \infty} \varphi\left(C^{i}\right)=\varphi(C)$.

As is well-known, continuity is equivalent to the conjunction of upper and lower semicontinuity.

Upper semicontinuity. For all $C \in \mathcal{C}$, for all sequences $\left\langle C^{i}\right\rangle_{i \in \mathbb{N}}$ with $C^{i} \in \mathcal{C}$ for all $i \in \mathbb{N}$, for all $x \in \mathbb{R}^{n}$ and for all sequences $\left\langle x^{i}\right\rangle_{i \in \mathbb{N}}$ with $x^{i} \in \varphi\left(C^{i}\right)$ for all $i \in \mathbb{N}$, if $\lim _{i \rightarrow \infty} x^{i}=x$, then $x \in \varphi(C)$.

Lower semicontinuity. For all $C \in \mathcal{C}$, for all sequences $\left\langle C^{i}\right\rangle_{i \in \mathbb{N}}$ with $C^{i} \in \mathcal{C}$ for all $i \in \mathbb{N}$ and for all $x \in \varphi(C)$, if $\lim _{i \rightarrow \infty} C^{i}=C$, then there exists a sequence $\left\langle x^{i}\right\rangle_{i \in \mathbb{N}}$ with $x^{i} \in \varphi\left(C^{i}\right)$ for all $i \in \mathbb{N}$ such that $\lim _{i \rightarrow \infty} x^{i}=x$.

Unlike in the single-plateaued case discussed in Bossert and Peters (2013), it would not make much sense to impose lower semicontinuity (and thus, a fortiori, continuity) in the single-basined setting. The reason is that lower semicontinuity would rule out even the most well-behaved single-basined choice correspondences. To see this, consider the following example. Suppose that $n=2$ and let $\varphi(C)=\arg \max _{x}\{d(x,(0,0)) \mid x \in C\}$ for all $C \in \mathcal{C}$. This is a single-basined (actually, single-dipped) choice correspondence with the 
basin $B=\{(0,0)\}$. Clearly, $\varphi$ is not lower semicontinuous (and, thus, not continuous) but it is upper semicontinuous.

As shown in the following theorem, a basin must be closed if the choice correspondence is to be upper semicontinuous in addition to satisfying independence of irrelevant alternatives and single-basinedness. This restriction cannot be obtained by means of the two original axioms alone; see the example following the statement and proof of the theorem.

Theorem 3 Let the choice correspondence $\varphi: \mathcal{C} \rightarrow \mathbb{R}^{n}$ satisfy independence of irrelevant alternatives, single-basinedness and upper semicontinuity, and suppose that $\varphi$ has a basin $B$. Then $B$ is closed.

Proof. If $B$ is a singleton, we are done. If not, let $x \in b d(B)$. We have to show that $x \in B$. Take $y \in B \backslash\{x\}$. Also, let $x^{1}, x^{2}, \ldots \in B$ be such that $\lim _{i \rightarrow \infty} x^{i}=x$. Since $B$ is convex by Lemma 4 , we have $\left[x^{i}, y\right] \subseteq B$ and thus $\varphi\left(\left[x^{i}, y\right]\right)=\left[x^{i}, y\right]$ for all $i \in \mathbb{N}$ by Theorem $1(\mathrm{a})$. Hence, $\varphi([x, y])=[x, y]$ by upper semicontinuity. By Theorem $1(\mathrm{~b})$, this implies $[x, y] \subseteq B$ and, in particular, $x \in B$.

That upper semicontinuity cannot be dispensed with in the above theorem is established by the following example. Let $n=2$ and let $B$ be the open disc with center $(0,0)$ and radius 1. Now define $\varphi(C)=C$ if $C \subseteq B$ and $\varphi(C)=\arg \max _{x}\{d(x,(0,0)) \mid x \in C\}$ otherwise. This is a choice correspondence with a unique convex basin $B$ that satisfies independence of irrelevant alternatives and single-basinedness but the basin clearly is not closed.

Also, the converse of Theorem 3 does not hold. The following is an example of a choice function with a closed (even compact) basin that satisfies independence of irrelevant alternatives and single-basinedness, but not upper semicontinuity. Let $n=2$ and let $\varphi$ pick the lexicographically maximal point among all points of a choice set $C$ that have maximal Euclidean distance to (the unique basin point) $(0,0)$.

\section{$7 \quad$ Representability}

A choice correspondence $\varphi: \mathcal{C} \rightarrow \mathbb{R}^{n}$ is rationalizable if there exists a transitive binary relation $R$ on $\mathbb{R}^{n}$ such that

$$
\varphi(C)=\{x \in C \mid x R y \text { for all } y \in C\}
$$

for all $C \in \mathcal{C}$. In this case we say that $R$ rationalizes $\varphi$. A choice function $\varphi$ is rationalizablerepresentable if there exists a transitive binary relation $R$ on $\mathbb{R}^{n}$ and a function $u: \mathbb{R}^{n} \rightarrow \mathbb{R}$ 
such that $R$ rationalizes $\varphi$ and

$$
[x R y \Rightarrow u(x) \geq u(y)] \text { and }[x P y \Rightarrow u(x)>u(y)]
$$

for all $x, y \in \mathbb{R}^{n}$, where $P$ is the asymmetric part of $R$. In this case, we say that $u$ represents $R$. The natural candidate for a rationalizing binary relation is the transitive closure of $R_{\varphi}$, that is, the indirect revealed preference relation $\overline{R_{\varphi}}$, defined by

$$
\begin{aligned}
x \overline{R_{\varphi}} y: \Leftrightarrow & \text { there are } m \in \mathbb{N} \backslash\{1\} \text { and } x^{1}, \ldots, x^{m} \in \mathbb{R}^{n} \text { such that } \\
& x=x^{1}, x^{i} R_{\varphi} x^{i+1} \text { for all } i \in\{1, \ldots, m-1\} \text { and } x^{m}=y
\end{aligned}
$$

for all $x, y \in \mathbb{R}^{n}$. Clearly, to use $\overline{R_{\varphi}}$ for rationalizability-representability of $\varphi$, a necessary condition is that $R_{\varphi}$ be Suzumura consistent; see Suzumura (1976) and Bossert and Suzumura (2010).

Suzumura consistency. For all $x, y \in \mathbb{R}^{n}$,

$$
x \overline{R_{\varphi}} y \Rightarrow \neg\left(y P_{\varphi} x\right) .
$$

Suzumura consistency is weaker than transitivity and stronger than acyclicity, which rules out cycles of strict preferences. In general, the conditions studied in this paper (namely, independence of irrelevant alternatives, single-basinedness and upper semicontinuity combined) are not sufficient to guarantee Suzumura consistency of $R_{\varphi}$. We do not go into this matter in more detail here but refer the reader to earlier work (Bossert and Peters, 2009, 2013) for the case of single-peaked and single-plateaued choice. We show that adding Suzumura consistency to the three above-mentioned conditions implies that $\varphi$ is rationalizablerepresentable. Thus, full continuity of $\varphi$ is not needed to obtain this result.

We start with the following auxiliary result. For $x \in \mathbb{R}^{n}$ and $\varepsilon>0$ we write $\mathbf{B}(x, \varepsilon)$ for the open (Euclidean) ball with center $x$ and radius $\varepsilon$.

Lemma 6 Let the choice correspondence $\varphi$ satisfy independence of irrelevant alternatives, single-basinedness and upper semicontinuity. Let $x, y \in \mathbb{R}^{n}$ be such that $x P_{\varphi} y$. Then there exists $a \in \mathbb{Q}^{n}$ such that $x P_{\varphi} a P_{\varphi} y$.

Proof. $x P_{\varphi} y$ and independence of irrelevant alternatives imply $x \in \varphi([x, y])$, hence $x R_{\varphi} z$ for all $z \in[x, y]$. Suppose that there exists $z \in(x, y)$ with $z R_{\varphi} x$. Then $y R_{\varphi} x$ by singlebasinedness, a contradiction. Hence $x P_{\varphi} z$ for all $z \in(x, y]$, and $\{x\}=\varphi([x, y])$. Now let $x^{k} \in(x, y]$ for all $k \in \mathbb{N}$ be such that $x^{k} \rightarrow x$ as $k \rightarrow \infty$. By upper semicontinuity, there 
exist $k \in \mathbb{N}$ and $z^{k} \in\left[x^{k}, y\right)$ such that $z^{k} \in \varphi\left(\left[x^{k}, y\right]\right)$ and $y \notin \varphi\left(\left[x^{k}, y\right]\right)$. By independence of irrelevant alternatives this implies $z^{k} P_{\varphi} y$. We conclude from these arguments that there exists $z \in(x, y)$ such that $x P_{\varphi} z P_{\varphi} y$.

Since $x P_{\varphi} z$, hence (by the same arguments as before) $\{x\}=\varphi([x, z])$, upper semicontinuity implies that there exists $\varepsilon_{1}>0$ such that for all $v \in \mathbf{B}\left(z, \varepsilon_{1}\right)$, we have $\varphi([x, v]) \cap$ $\mathbf{B}\left(z, \varepsilon_{1}\right)=\emptyset$. Let $v \in \mathbf{B}\left(z, \varepsilon_{1}\right)$ and $v^{\prime} \in \varphi([x, v])$. Then $v^{\prime} P_{\varphi} v$. By single-basinedness, $x R_{\varphi} v$, and since $v \notin \varphi([x, v])$, independence of irrelevant alternatives implies $x P_{\varphi} v$.

Since $z P_{\varphi} y$, hence (by the same arguments as before) $\{z\}=\varphi([z, y])$, there exists, again by upper semicontinuity, $\varepsilon_{2}>0$ such that $y \notin \varphi([v, y])$ for all $v \in \mathbf{B}\left(z, \varepsilon_{2}\right)$. For any such $v$, let $v^{\prime} \in \varphi([v, y])$. Then by independence of irrelevant alternatives, $v^{\prime} P_{\varphi} y$, and hence by single-basinedness and the fact that $y \notin \varphi([v, y])$, we obtain $v P_{\varphi} y$.

Let $\varepsilon=\min \left\{\varepsilon_{1}, \varepsilon_{2}\right\}$. Take $a \in \mathbf{B}(z, \varepsilon) \cap \mathbb{Q}^{n}$. Then, by the preceding paragraphs, $x P_{\varphi} a P_{\varphi} y$.

The rationalizability-representability result is the following.

Theorem 4 Let the choice correspondence $\varphi$ satisfy independence of irrelevant alternatives, single-basinedness and upper semicontinuity. Let $R_{\varphi}$ be Suzumura consistent. Then $\overline{R_{\varphi}}$ rationalizes $\varphi$ and $\varphi$ is rationalizable-representable.

Proof. Let $C \in \mathcal{C}$ and $x \in \varphi(C)$. Then $x R_{\varphi} y$ for all $y \in C$, so in particular $x \overline{R_{\varphi}} y$ for all $y \in C$. Conversely, let $x \overline{R_{\varphi}} y$ for all $y \in C$. Suppose $x \notin \varphi(C)$. Let $y \in \varphi(C)$. By independence of irrelevant alternatives, we have $x \overline{R_{\varphi}} y$ and $y P_{\varphi} x$. This, however, contradicts Suzumura consistency of $R_{\varphi}$. Hence, $\overline{R_{\varphi}}$ rationalizes $\varphi$.

Let $x, y \in \mathbb{R}^{n}$ with $x \neq y$. Let $\overline{I_{\varphi}}$ and $\overline{P_{\varphi}}$ denote the symmetric and asymmetric parts of $\overline{R_{\varphi}}$, respectively. If $x \overline{I_{\varphi}} y$, then clearly, for all $a \in \mathbb{Q}^{n}$, we have $a \overline{R_{\varphi}} x \Leftrightarrow a \overline{R_{\varphi}} y$ and $x \overline{R_{\varphi}} a \Leftrightarrow y \overline{R_{\varphi}} a$. If $x \overline{P_{\varphi}} y$, then Lemma 6 implies that there exists $a \in \mathbb{Q}^{n}$ such that $x \overline{P_{\varphi}} a \overline{P_{\varphi}} y$.

Now let $\mathbb{Q}^{n}=\left\{a_{1}, a_{2}, \ldots\right\}$ be an enumeration of the countable set $\mathbb{Q}^{n}$. We define $u: \mathbb{R}^{n} \rightarrow \mathbb{R}$ by

$$
u(x)=\sum_{\left\{k \in \mathbb{N} \mid x \overline{R_{\varphi}} a_{k}\right\}} 2^{-k}
$$

for all $x \in \mathbb{R}^{n}$. By the preceding paragraph and by transitivity of $\overline{R_{\varphi}}, u$ represents $\overline{R_{\varphi}}$.

The construction of $u$ in the proof of Theorem 4 is familiar from Debreu (1954) and Jaffray (1975). 
Furthermore, it can be shown that $u$ is quasi-convex. Let the choice function $\varphi$ satisfy independence of irrelevant alternatives and single-basinedness. Let $\varphi$ be rationalized by $\overline{R_{\varphi}}$, and let $\overline{R_{\varphi}}$ be represented by $u: \mathbb{R}^{n} \rightarrow \mathbb{R}$. Let $x, y \in \mathbb{R}^{n}$ and $z \in(x, y)$. If $x \in \varphi([x, y])$ then $u(x) \geq u(z)$, so that $u(z) \leq \max \{u(x), u(y)\}$. Similarly, if $y \in \varphi([x, y])$ then $u(y) \geq u(z)$, so that again $u(z) \leq \max \{u(x), u(y)\}$. If $z^{\prime} \in \varphi([x, y])$ for some $z^{\prime} \in(x, y)$, then by Lemma 3 , $[x, y]=\varphi([x, y])$, so that $u(z)=u(x)=u(y)=\max \{u(x), u(y)\}$. Hence, $u$ is quasi-convex.

\section{References}

Arrow, K. (1959). Rational choice functions and orderings. Economica 26, 121-127.

Ballester, M. and G. Haeringer (2011). A characterization of the single-peaked domain. Social Choice and Welfare 36, 305-322.

Barberà, S. (2007). Indifferences and domain restrictions. Analyse \& Kritik 29, 146-162.

Barberà, S., D. Berga and B. Moreno (2012). Domains, ranges and strategy-proofness: the case of single-dipped preferences. Social Choice and Welfare 39, 335-352.

Barberà, S., F. Gul and E. Stacchetti (1993). Generalized median voting schemes and committees. Journal of Economic Theory 61, 262-289.

Barberà, S. and M. Jackson (1994). A characterization of strategy-proof social choice functions for economies with pure public goods. Social Choice and Welfare 11, 241252.

Berga, D. (1998). Strategy-proofness and single-plateaued preferences. Mathematical Social Sciences 35, 105-120.

Berga, D. and B. Moreno (2009). Strategic requirements with indifference: single-peaked versus single-plateaued preferences. Social Choice and Welfare 32, 275-298.

Besfamille, M. and J. Lozachmeur (2010). NIMBY and mechanism design under different constitutional constraints. International Tax and Public Finance 17, 114-132.

Black, D. (1948). On the rationale of group decision-making. Journal of Political Economy $56,23-34$.

Bossert, W. and H. Peters (2009). Single-peaked choice. Economic Theory 41, 213-230. 
Bossert, W. and H. Peters (2013). Single-plateaued choice. Mathematical Social Sciences, forthcoming.

Bossert, W. and K. Suzumura (2010). Consistency, Choice, and Rationality. Cambridge, MA: Harvard University Press.

Debreu, G. (1954). Representation of a preference ordering by a numerical function. In: Thrall, R., C. Coombs and R. Davies (eds.). Decision Processes, pp. 159-175. New York: Wiley.

Dutta, B., H. Peters and A. Sen (2002). Strategy-proof probabilistic mechanisms in economies with pure public goods. Journal of Economic Theory 106, 392-416.

Ehlers, L. (2002a). Strategy-proof allocation when preferences are single-plateaued. Review of Economic Design 7, 105-115.

Ehlers, L. (2002b). Probabilistic allocation rules and single-dipped preferences. Social Choice and Welfare 19, 325-348.

Ehlers, L. and T. Storcken (2008). Arrow's possibility theorem for one-dimensional singlepeaked preferences. Games and Economic Behavior 64, 533-547.

Hansson, B. (1968). Choice structures and preference relations. Synthese 18, 443-458.

Inada, K. (1969). The simple majority rule. Econometrica 37, 490-506.

Jaffray, J.-Y. (1975). Semicontinuous extension of a partial order. Journal of Mathematical Economics 2, 395-406.

Klaus, B. (2001). Coalitional strategy-proofness in economies with single-dipped preferences and the assignment of an indivisible object. Games and Economic Behavior 34, 64-82.

Klaus, B., H. Peters and T. Storcken (1997). Strategy-proof division of a private good when preferences are single-dipped. Economic Letters 55, 339-346.

Kunreuther, H. and P. Kleindorfer (1986). A sealed-bid auction mechanism for siting noxious facilities. American Economic Review (Papers and Proceedings) 76, 295299. 
Le Breton, M. and J. Weymark (2011). Arrovian social choice theory on economic domains. In: K. Arrow, A. Sen and K. Suzumura (eds.). Handbook of Social Choice and Welfare, vol. 2, pp. 191-299. Amsterdam: Elsevier.

Lescop, D. (2007). Optimal mechanisms for siting noxious facilities. Review of Economic Design 10, 273-284.

Manjunath, V. (2013). Efficient and strategy-proof social choice when preferences are single-dipped. International Journal of Game Theory, forthcoming.

Moulin, H. (1980). On strategy-proofness and single-peakedness. Public Choice 35, 437455.

Moulin, H. (1984). Generalized Condorcet-winners for single peaked and single-plateau preferences. Social Choice and Welfare 1, 127-147.

Nash, J. (1950). The bargaining problem. Econometrica 18, 155-162.

Öztürk, M., H. Peters and T. Storcken (2012). On the location of public bads: strategyproofness under two-dimensional single-dipped preferences. METEOR Research Memorandum 12/040, Maastricht.

Öztürk, M., H. Peters and T. Storcken (2013). Strategy-proof location of a public bad on a disc. Economics Letters 119, 14-16.

Peremans, W. and T. Storcken (1999). Strategy-proofness on single-dipped preference domains. In: H. de Swart (ed.). Logic, Game Theory and Social Choice, 296-313. Tilburg: Tilburg University Press.

Richter, M. (1966). Revealed preference theory. Econometrica 41, 1075-1091.

Richter, M. (1971). Rational choice. In: J. Chipman, L. Hurwicz, M. Richter and H. Sonnenschein (eds.). Preferences, Utility, and Demand, pp. 29-58. New York: Harcourt Brace Jovanovich.

Samuelson, P. (1938). A note on the pure theory of consumer's behaviour. Economica 5, $61-71$.

Sen, A. (1970). Collective Choice and Social Welfare. San Francisco: Holden-Day.

Shubik, M. (1982). Game Theory in the Social Sciences: Concepts and Solutions. Cambridge, MA: MIT Press. 
Sprumont, Y. (1991). The division problem with single-peaked preferences: a characterization of the uniform allocation rule. Econometrica 59, 509-519.

Suzumura, K. (1976). Remarks on the theory of collective choice. Economica 43, 381-390. 